\title{
INVESTIGACIONES
}

\section{Cultura institucional de promoción de estudios universitarios y proceso de elección de estudios}

\author{
Institutional college-going culture and college choice process
}

\author{
Álvaro González Sanzana \\ Facultad de Educación, Pontificia Universidad Católica de Chile \\ Telf.: (2) 242073373. Correo electrónico: algonzalezs@uc.cl
}

\begin{abstract}
RESUMEN
A través de entrevistas realizadas a diferentes actores claves de cuatro establecimientos secundarios, este artículo explora la presencia e influencia de una cultura institucional de ir a la universidad en los colegios sobre el proceso de elección de estudios por parte de los alumnos. La cultura institucional de ir a la universidad es entendida en este estudio como la combinación del habitus institucional y de las estrategias de promoción de estudios universitarios por parte del colegio. Se evalúa la influencia de la cultura institucional de ir a la universidad a través del modelo de elección de estudios en tres etapas de Hossler y Gallagher (1987). Los resultados indican que la presencia o ausencia de una cultura institucional de ir a la universidad conforma un conjunto de oportunidades que puede ampliar o limitar el horizonte de posibilidades de estudio consideradas por un alumno al finalizar la enseñanza secundaria.
\end{abstract}

Palabras clave: enseñanza secundaria, enseñanza superior, orientación postsecundaria, elección de estudios.

\begin{abstract}
The following paper examines the existence of a "go-to university" institutional culture in secondary schools and its influence over the students' academic enrolment in selective universities. In the present research, the "go-to university" institutional culture is understood as the combination of the institutional habitus and the promotion of tertiary studies carried out by schools. In order to fulfill the objectives, a survey was conducted among key actors of four different schools with dissimilar socioeconomic status based in the province of Concepcion, Chile. We assessed the impact of this institutional culture through the Hossler and Gallagher (1987) three-phase college choice model (predisposition, search and choice). The results indicate that the existence or absence of a "go-to university" institutional culture represents a series of opportunities that can either widen or limit the range of possibilities concerning the students' decision-making process.
\end{abstract}

Key words: secondary education, higher education, post high school guidance, choice of studies. 


\section{INTRODUCCIÓN}

A nivel internacional, no existen muchas investigaciones en relación a la influencia de los colegios sobre las orientaciones académicas y la elección de estudios superiores por parte de los alumnos. En este estudio se aborda la influencia del establecimiento educacional secundario sobre el destino de sus alumnos en la enseñanza superior — particularmente la universidad - a través de la cultura institucional de ir a la universidad, y se evalúa su influencia en los alumnos a través de las tres etapas del proceso de elección de estudios superiores definidas por Hossler y Gallagher (1987): la predisposición, la búsqueda de información y la elección final.

En esta investigación se entiende la cultura institucional de ir a la universidad por parte de los establecimientos secundarios como una noción que se construye en torno a dos conceptos: el habitus institucional y las estrategias de promoción de estudios universitarios.

El habitus institucional y las estrategias de promoción de estudios universitarios lo que se ha denominado en este estudio "cultura institucional de ir a la universidad"forman un conjunto de características estructurales y culturales de un colegio que tienen un fuerte impacto sobre el destino postsecundario de los alumnos, ya que se encargan de ampliar (en caso de presencia) o limitar (en caso de ausencia) el horizonte de posibilidades de estudios futuros. En efecto, los alumnos toman decisiones en un contexto de mensajes implícitos y explícitos vehiculados por su entorno social, familiar y escolar (Hossler, Schmit y Vesper, 1999; McDonough, 1997). Este artículo se centra en entender el rol jugado por el establecimiento educacional en este proceso.

Se ha optado, dentro de la multitud de modelos de elección de estudios existente en la literatura especializada, por el modelo de los autores Hossler y Gallagher (1987), pues ha sido ampliamente utilizado en investigaciones relativas al proceso de elección de estudios, principalmente en países anglosajones (Perna, 2006), pero nunca en Chile.

Para aprehender cómo se manifiestan las culturas institucionales en cada una de las etapas del modelo de elección de estudios, se ha recurrido, por un lado, a un análisis de documentos oficiales emanados de los propios colegios y, por otro lado, al discurso de apoderados, directivos, docentes y alumnos, relativos a las estrategias institucionales de los establecimientos secundarios en torno a los estudios universitarios (currículum, preparación para la PSU, etc.) y los valores y prácticas vehiculados en cuatro establecimientos educacionales científico-humanistas seleccionados para este estudio.

El artículo se presenta de la manera siguiente. La primera parte, teórica, aborda la forma en que se entiende la cultura institucional de ir a la universidad, y presenta el modelo de elección de estudios en tres etapas. La segunda parte explica la metodología empleada en esta investigación, de corte cualitativo, detallando la muestra con la que se trabaja y los métodos de recolección y análisis de la información. A continuación se presenta un estudio comparado de la influencia de las culturas institucionales de cuatro establecimientos secundarios en torno al proceso de elección de estudios universitarios por parte de los alumnos. Finalmente, se termina por una discusión de las implicancias de los resultados para la comprensión de este proceso. 


\section{REVISIÓN DE LA LITERATURA}

\subsection{LA MODELIZACIÓN DEL PROCESO DE ELECCIÓN DE ESTUDIOS}

En la década de 1980, principalmente en los Estados Unidos, se sucedieron una serie de modelos que intentaban explicar de manera lo más completa posible el proceso de elección de estudios superiores por parte de los alumnos, acordando cada uno de ellos diferentes grados de importancia al efecto del establecimiento escolar sobre este proceso (Chapman, 1981; Hossler y Gallagher, 1987; Jackson, 1982; Litten, 1982). Para todos estos autores, el proceso de elección de estudios universitarios es una combinación de variables, entre las que destacan el capital económico y cultural familiar, la capacidad académica del alumno, variables psicológicas — tales como la autoestima o el sentimiento de competencia-, las políticas públicas específicas a cada realidad nacional, las características de las universidades y, por supuesto, el contexto escolar. Más recientemente, otros autores han propuesto modelos que incorporan variables contextuales diversas para explicar el proceso de elección de estudios que, ha quedado claramente establecido, es multidimensional y extremadamente complejo (Perna, 2006).

En este estudio se utiliza el modelo de los investigadores norteamericanos Hossler y Gallagher (1987), quienes proponen un modelo en tres etapas. La primera etapa se denomina "predisposición". En esta, el alumno toma la decisión de continuar estudios superiores o tomar caminos alternativos, como integrarse directamente al mercado laboral o incorporarse a instituciones como las fuerzas armadas, por ejemplo. Los planes de estudios post-secundarios, en esta etapa, se ven altamente influenciados por las familias del alumno, pero también por su contexto escolar (Hossler y Gallagher, 1987). En relación al establecimiento de educación secundaria, serán fundamentales en esta etapa la calidad de la educación recibida, el plan de estudios o currículum del colegio, así como el apoyo y estímulo recibido por los alumnos de parte de los profesores (Hossler y Stage, 1992). Para estos autores se distinguen en esta fase tres tipos de estudiantes: los alumnos que consideran varias universidades como opción de estudios, aquellos que ya saben que solo pueden acceder a una o dos universidades (o tipos de universidades) y los que no tienen intención de proseguir estudios universitarios.

La segunda etapa del modelo se denomina "búsqueda de información”. Es el proceso por el cual el alumno se informa sobre instituciones específicas y sus características. Implica el descubrimiento y evaluación por parte de los alumnos del universo de instituciones disponibles en las que, en principio, podrían inscribirse. Para Hossler et al. (1999), esta etapa es la más importante y la que mejor se presta para una intervención de parte de los establecimientos secundarios y de las universidades. Es una etapa bisagra de incertidumbre y de exploración en la que el rol del colegio es, una vez más, de gran importancia, tal como lo demostraron Plank y Jordan (2001). Estos autores concluyeron de un estudio realizado en los Estados Unidos que una gran cantidad de alumnos destacados, principalmente de condición socioeconómica desfavorecida, no iban a la universidad debido a un proceso deficiente de orientación e información por parte de los colegios. A la inversa, cuando los establecimientos secundarios se movilizan en torno a ciertas prácticas activas de información y orientación, las probabilidades de un individuo de seguir estudios universitarios aumentaban.

La tercera y última etapa es la "elección final", el momento en el que los alumnos eligen una institución específica para postular a ella. Las condiciones de admisión de cada 
universidad, los planes de ayuda financiera y, por supuesto, en un contexto de admisión a la universidad como el chileno, los resultados en la prueba de selección universitaria (PSU), serán decisivos. Por lo demás, en un país en el que las instituciones de enseñanza superior reproducen en gran parte la composición socioeconómica de los colegios, es factible imaginar que la tendencia de los estudiantes será sentirse atraído por una institución que alberga a individuos similares a ellos. Es, a priori, la etapa en la que el establecimiento escolar juega un rol menos preponderante.

\subsection{LA CULTURA INSTITUCIONAL DE PROMOCIÓN DE ESTUDIOS UNIVERSITARIOS O “CULTURA DE IR A LA UNIVERSIDAD"}

Existe una amplia literatura relativa a las culturas institucionales de los establecimientos escolares, la cual no es abordada en esta revisión (para una profundización del concepto, su origen y evolución, ver Draelants y Dumay, 2011). Este estudio se centra de manera específica, en lo que McDonough (1997) define como "cultura de ir a la universidad", es decir, la conjunción de una serie de elementos relativos principalmente a la cultura y a los valores vehiculados por la institución, pero también al currículum académico del colegio, la promoción de un clima especial y altas expectativas de parte de la comunidad escolar sobre el proceso de elección de estudios.

En efecto, la literatura ha mostrado que existen influencias específicas ligadas al establecimiento educacional sobre las aspiraciones de estudios superiores de los alumnos y su destino posterior (Draelants y Ardoisenet, 2011; Hossler et al., 1999; McDonough, 1997; Perna, 2006; Reay, David y Ball, 2001). Para explorar estas influencias específicas del establecimiento escolar, se ha decidido agrupar bajo la noción de "cultura de ir a la universidad" una definición un poco más amplia de la considerada originalmente por McDonough (1997). Por un lado, se retoma el concepto de habitus institucional (McDonough, 1997; Reay et al., 2001) y, por otro lado, las estrategias de promoción de estudios superiores a través de las normas y recursos organizacionales (Draelants y Ardoisenet, 2011; Hill, 2008). Esta combinación de conceptos permite dar cuenta tanto de percepciones y discursos subjetivos, permeadas por el entorno socioeconómico y cultural, como de estrategias intencionadas y racionales de parte de los establecimientos educacionales.

\subsubsection{El habitus institucional}

El primer componente de una cultura de ir a la universidad es el habitus institucional. Según Reay et al. (2001), esta noción es útil para aprehender el rol de la escuela sobre el destino educacional de los alumnos, ya que constituye una compleja amalgama entre agencia y estructura y podría entenderse como el impacto de un grupo cultural o clase social sobre el comportamiento de un individuo, mediado por una organización. Estos autores argumentan que los colegios se encuentran vinculados a contextos socioculturales más amplios a través de procesos en los que ambos se influencian mutuamente.

Una de las autoras que más ha trabajado este concepto es McDonough (1997). Según ella, el habitus corresponde a un conjunto común de percepciones subjetivas asumidas por los miembros de un grupo o una clase social, que influencia las aspiraciones y actitudes de un individuo. Estas aspiraciones son, a la vez, evaluaciones subjetivas de las posibilidades futuras y probabilidades objetivas. No son análisis racionales, sino más bien la manera en 
la que los alumnos que provienen de clases sociales diferentes toman decisiones razonables y juiciosas para sus propias aspiraciones. El habitus institucional influirá fuertemente, señala, en limitar o expandir el universo de posibilidades de los alumnos. Para McDonough (1997), la disparidad de misiones, de la organización y de recursos destinados a los servicios de orientación en los colegios tiene necesariamente un impacto sobre las aspiraciones y decisiones de los alumnos ${ }^{1}$.

Cabe destacar, sin embargo, el efecto diferenciado del habitus institucional sobre los alumnos, ya que los individuos se encuentran desigualmente posicionados en relación al habitus institucional del colegio, en la medida en que las influencias de la familia y el grupo de pares sea concordante o discordante con aquellas ejercidas por la institución ${ }^{2}$.

\subsubsection{Las estrategias de promoción de estudios universitarios}

El segundo componente de una cultura institucional de ir a la universidad es un conjunto intencionado de estrategias de promoción de los estudios universitarios, caracterizado por algunos autores, como Hill (2008) y Draelants y Ardoisenet (2011), por dos dimensiones: los recursos formales que el establecimiento pone a disposición de los alumnos (tales como la malla curricular, la preparación académica, la existencia de cursos de preparación específicos para la universidad, etc.) y las normas organizacionales de la institución (referidas a los valores y las prácticas vehiculados por el establecimiento).

De esta manera y a modo de resumen, este estudio retiene como definición de cultura de ir a la universidad una amalgama entre el habitus institucional y las estrategias de promoción de los estudios universitarios, que van a permitir a los alumnos plantearse (o no) la posibilidad de proseguir carreras universitarias.

\section{OBJETIVO E HIPÓTESIS}

El presente estudio tiene por objetivo analizar en qué medida la presencia o ausencia de una cultura de ir a la universidad en el establecimiento secundario influencia las aspiraciones y decisiones de los alumnos en cada una de las etapas del proceso de elección de estudios — predisposición, búsqueda de información y elección—, con la finalidad de identificar la naturaleza de esta influencia y sus características.

En torno a este objetivo, se plantean dos hipótesis centrales:

a. La presencia o ausencia de una cultura de ir a la universidad en el establecimiento secundario puede limitar o ampliar el universo de posibilidades de elección de estudios superiores de un alumno.

b. La cultura de ir a la universidad en un establecimiento escolar influencia a los alumnos con más claridad en las etapas de predisposición y búsqueda de información que en la etapa de elección final.

Para el contexto chileno, revisar el estudio de Lagos y Palacios (2008) sobre orientación vocacional y profesional en colegios de bajo nivel socioeconómico.

2 No todos los autores coinciden en la pertinencia de utilizar el concepto de habitus para las instituciones u organizaciones educativas. Al respecto, ver el debate con las críticas de Atkinson (2011) y la respuesta de Burke, Emmerich e Ingram (2013) en British Journal of Sociology of Education. 


\section{METODOLOGÍA}

\subsection{PARTICIPANTES}

Este estudio se inscribe en el marco de una investigación más amplia cuya primera etapa se llevó a cabo en una muestra representativa de estudiantes de último año de enseñanza secundaria pertenecientes a 23 establecimientos de modalidad científico-humanista de la provincia de Concepción $(\mathrm{N}=6931 ; \mathrm{n}=1054)$. La primera etapa de esta investigación, cuyo enfoque de investigación fue cuantitativo, tenía por objetivo general entender cuáles son los factores individuales y colectivos que influencian las aspiraciones a estudiar en la universidad y concretizar esas aspiraciones.

Para la segunda parte de esta investigación, y este estudio en particular, se ha decidido seleccionar cuatro colegios. Como el objetivo es comprender la influencia de la cultura institucional sobre el proceso de elección de estudios superiores, se ha elegido cuatro colegios que puedan ser contrastados desde el punto de vista de las estrategias institucionales, normas y valores relativos a la promoción de estudios universitarios en sus alumnos.

Para elegir a estos colegios, se ha recurrido a una encuesta original — que forma parte de la primera parte de esta investigación — realizada en octubre de 2012 en 23 establecimientos educacionales. Los resultados de esa encuesta permitieron identificar cuatro colegios de características sensiblemente diferentes en relación a los recursos formales y normas organizacionales destinados a la promoción de los estudios universitarios. Además, estos establecimientos se distinguen en cuanto a la composición socioeconómica de sus estudiantes y el nivel académico promedio (medido a través de los resultados en la Prueba de Selección Universitaria). En total, 216 alumnos de estos cuatro colegios respondieron a esta encuesta, durante la primera fase de la investigación.

Un año después de aplicada la encuesta, se ha contactado a los colegios y realizado en ellos un total de 37 entrevistas con los directores, orientadores, directores académicos, centros de padres, centros de alumnos, exalumnos y profesores jefes (que tuviesen más de diez años de experiencia docente en el colegio), durante los meses de septiembre y octubre de 2013.

\subsection{RECOLECCIÓN Y ANÁLISIS DE LA INFORMACIÓN}

La recolección de información se desarrolló en dos etapas. En primer lugar, se ha realizado un análisis de los documentos oficiales facilitados por los colegios y/o disponibles en las páginas web institucionales, tales como la reseña histórica del colegio, las características generales de este, el proyecto educativo institucional, las funciones y objetivos del departamento de orientación, entre otros.

En una segunda etapa, se realizaron entrevistas de carácter semi-estructuradas a miembros del equipo directivo, orientadores, representantes del centro de padres, profesores jefes de cursos superiores, representantes del centro de alumnos y exalumnos de cada colegio.

En primer lugar, se contactó a los directores de colegio, quienes aceptaron que se realizara este estudio en las instituciones que dirigen. Posteriormente, una vez en el colegio, y habiendo realizado la entrevista al director (o directora), se procedió a contactar personalmente al resto de los actores descritos anteriormente. Por otro lado y de manera 
paralela, se tomó contacto con los exalumnos de los colegios seleccionados, a través de llamados telefónicos o envío de correos electrónicos.

Las entrevistas con los exalumnos de los colegios se realizaron en sus casas o en sus lugares de estudio y tenían por finalidad conocer las características del hogar del cual provienen, su recorrido escolar, los motivos por los que eligieron un plan diferenciado a finales de segundo año de enseñanza secundaria, conocer la caracterización que realizan del entorno escolar en el que se desenvolvieron, cuáles eran sus aspiraciones de estudios superiores, sus percepciones sobre las estrategias institucionales desplegadas durante el proceso de elección de estudios y las razones de su decisión final en relación a los estudios superiores.

Las entrevistas con miembros del equipo directivo, orientadores, centros de padres, centros de alumnos y profesores jefes se realizaron íntegramente en los colegios respectivos. Estas entrevistas tenían por objetivo indagar sobre los recursos formales (planes diferenciados, preparación para la PSU, preuniversitario, preparación para los estudios universitarios, contexto socioeconómico y cultural del alumnado, características del cuerpo docente, proceso de orientación e información, vínculos con universidades) y las normas organizacionales (valores y prácticas del establecimiento, proyección hacia los estudios superiores, apoyo a la realización de estudios universitarios) desplegadas por la institución en el proceso de elección de estudios.

Todas las entrevistas fueron grabadas en registro de audio, transcritas de manera integral y analizadas a través del software NVIVO 10. Se realizó, en primer lugar, una codificación general para organizar el material en áreas amplias de temas y a continuación se exploraron nodos para cada tema, con una codificación más detallada, hasta alcanzar el punto de saturación.

\section{RESULTADOS}

\subsection{CARACTERIZACIÓN DE LOS COLEGIOS}

Los colegios escogidos para este estudio se diferencian nítidamente entre sí, tanto en relación a su composición socioeconómica, como a los resultados de sus alumnos en la prueba de selección universitaria (PSU) y las estrategias institucionales desarrolladas para promover el acceso de los alumnos a la universidad.

A continuación se realiza una breve reseña de cada uno de estos establecimientos y se los compara (ver Tabla 1) en relación a una serie de tópicos relevantes para nuestro estudio ${ }^{3}$.

\section{Colegio A}

Este colegio es un establecimiento particular pagado, laico, trilingüe, mixto y que cuenta con subvención financiera y apoyo pedagógico de parte de un país europeo. Tradicionalmente asociado a una alta exigencia académica, se define como un colegio de

Debido a que nos comprometimos a mantener el anonimato, tanto de los entrevistados como de los establecimientos educacionales, es que nos referimos a estos últimos con las letras A, B, C y D. 
excelencia y bicultural. Los ejes de su proyecto educativo son el dominio de la lengua y el razonamiento, desarrollar el gusto y la práctica de la lectura, favorecer valores y competencias tales como el civismo, la autonomía y la responsabilidad, así como profundizar la formación integral a través del deporte, la cultura y la apertura internacional.

\section{Colegio B}

El colegio B corresponde a un establecimiento particular subvencionado tradicional, mixto, perteneciente a una congregación religiosa. Este colegio ha destacado en los últimos 10 años por aumentar en forma notable el rendimiento de sus egresados en la PSU y, por lo tanto, el ingreso de estos a las universidades. Es tal vez uno de los pocos colegios de la provincia en los que se genera mayor mixidad social, puesto que cobra un copago diferenciado por ingresos familiares y atiende a una proporción importante de alumnos de condición socioeconómica vulnerable, producto de una política intencionada de parte de la dirección del establecimiento, para lo cual dispone de becas de estudio.

\section{Colegio C}

Este establecimiento particular subvencionado tiene un origen más reciente, es mixto, pertenece a una congregación religiosa diferente a la del colegio $\mathrm{B}$, y sus principios orientadores — señalados en su proyecto educativo institucional- son una educación humanista y cristiana católica, que busca el desarrollo armónico de la persona y que la prepara para su proyecto de vida. Toda la formación de los alumnos de este colegio se encuentra fuertemente impregnada de un espíritu eucarístico. En el proyecto institucional del colegio se presenta como uno de los objetivos más relevantes del establecimiento hacer del alumno un cristiano comprometido y activo.

\section{Colegio D}

Este colegio es un establecimiento municipal centenario, es mixto y corresponde al liceo científico-humanista público por excelencia de su comuna. Desde el año 1994 ha participado en gran cantidad de programas de mejoramiento generados por el Ministerio de Educación. A pesar de ello, ha sufrido, al igual que la mayoría de los liceos municipales, una sensible fuga de estudiantes en los últimos años. Se define como un liceo científicohumanista pluralista y tolerante, laico, cuyo concepto de hombre es democrático y de respeto por la diversidad. Su desafío es entregar a sus estudiantes una formación académica de calidad que les asegure el éxito en la continuación de estudios superiores y en la formación para la vida, para convertirse en una alternativa real de continuación de estudios para la comunidad local. 
Tabla 1. Características generales de los colegios seleccionados

\begin{tabular}{|l|c|c|c|c|}
\hline & $\begin{array}{c}\text { Colegio } \\
\text { A }\end{array}$ & $\begin{array}{c}\text { Colegio } \\
\text { B }\end{array}$ & $\begin{array}{c}\text { Colegio } \\
\text { C }\end{array}$ & $\begin{array}{c}\text { Colegio } \\
\text { D }\end{array}$ \\
\hline $\begin{array}{l}\text { Dependencia } \\
\text { administrativa }\end{array}$ & Particular pagado & $\begin{array}{c}\text { Particular } \\
\text { Subvencionado }\end{array}$ & $\begin{array}{c}\text { Particular } \\
\text { Subvencionado }\end{array}$ & Municipal \\
\hline Nivel Socioeconómico & Alto & Medio-alto & Medio & Medio-bajo \\
\hline Vulnerabilidad escolar & $0.0 \%$ & $0.0 \%>23 \%$ & $23.01 \%>40.0 \%$ & $40,01 \%>61,0 \%$ \\
\hline Orientación & Laica & Católica & Católica & Laica \\
\hline Provincia & Concepción & Concepción & Concepción & Concepción \\
\hline Área & Urbana & Urbana & Urbana & Urbana \\
\hline Promedio PSU 2012 & $>630$ & $\begin{array}{c}>600 \\
<630\end{array}$ & $<500$ & $<500$ \\
\hline $\begin{array}{l}\text { Opciones de planes } \\
\text { diferenciados }\end{array}$ & 6 & 3 & 3 & 0 \\
\hline
\end{tabular}

Como se puede observar en la Tabla 1, existe una relación directa entre el nivel socioeconómico del colegio y los resultados de los alumnos en la prueba de selección universitaria (PSU). Más llamativo es el hecho de que los planes diferenciados, que forman parte de los planes y programas del Ministerio de Educación chileno, no sean ofrecidos en todos los colegios. En nuestra muestra, el colegio de dependencia municipal no ofrece ningún plan diferenciado a sus alumnos de tercer y cuarto año de enseñanza secundaria, en tanto los colegios particulares subvencionados ofrecen tres alternativas y el colegio particular pagado seis opciones. La justificación de este último colegio está basada en su proyecto educativo de educación intercultural bilingüe, que le lleva a ofrecer más alternativas de las que propone el Ministerio de Educación. En tanto, en el liceo municipal explican la decisión de no ofrecer planes diferenciados por la escasez de profesores especialistas y la dificultad práctica de organizar dichos programas. Esta comparación permite un primer acercamiento en relación a las posibilidades que pueda ofrecer un colegio y otro en torno a la apertura de horizontes de estudios.

El índice de vulnerabilidad escolar fue obtenido a través de los resultados SIMCE (sistema nacional de evaluación de resultados de aprendizaje) y refleja la condición de riesgo asociada a los estudiantes de cada establecimiento. Se presenta en forma de porcentaje la vulnerabilidad del establecimiento en base a cinco prioridades excluyentes, calculadas por la JUNAEB (Junta Nacional de Auxilio Escolar y Becas). 
Estudios Pedagógicos XLII, Nº 3: 171-189, 2016

CULTURA INSTITUCIONAL DE PROMOCIÓN DE ESTUDIOS UNIVERSITARIOS Y PROCESO DE ELECCIÓN DE ESTUDIOS

\subsection{EL PROCESO DE ELECCIÓN DE ESTUDIOS}

\subsubsection{La fase de predisposición}

\subsubsection{La influencia de las familias sobre las aspiraciones}

Para los alumnos del colegio A, la universidad es una vía natural de prosecución de estudios después de la enseñanza secundaria, fundamentalmente porque provienen de hogares en los que los padres son casi exclusivamente universitarios, y son muy pocos los alumnos que desean o se atreven a plantear la posibilidad de seguir estudios en instituciones superiores no universitarias:

"Nos educaron desde pequeños que la universidad era lo que había después del colegio, no había otra opción, obligatoriamente, no era algo optativo”. (Exalumno colegio A).

Los alumnos del colegio B siguen el mismo patrón de conducta. Conscientes de pertenecer a un colegio de alto rendimiento académico y de la posibilidad, para muchos de ellos, de pasar a ser la primera generación universitaria de la familia, la universidad aparece como el camino lógico a seguir:

"Tenemos el concepto de que ir a un Instituto Profesional es cuando no te da el puntaje. Todos aspiramos a obtener un buen puntaje y quedar en una universidad tradicional. Mi mamá siempre me ha dicho que es porque no quiere que me pase lo mismo que a ella. Ella me dice que para que yo pueda vivir bien debo estudiar en la universidad". (Presidente del Centro de Alumnos colegio B).

En el colegio C, las aspiraciones de los alumnos se decantan ampliamente por los estudios universitarios, pero, a diferencia de los colegios anteriores, se aprecia una mayor dispersión de estas y la posibilidad abierta de optar por alternativas a la educación universitaria. Sin embargo, las familias incentivan mayoritariamente un camino universitario.

El colegio D, finalmente, muestra perfiles de familias muy heterogéneos, desde aquellas que motivan a sus hijos a seguir estudios universitarios bajo la lógica de acceder a un nivel de vida más elevado que el que los padres tuvieron, hasta aquellas que no los incentivan o derechamente los ven como una alternativa poco realista para sus hijos.

\subsubsection{La influencia del colegio sobre las aspiraciones}

Las instituciones educativas seleccionadas, en sus proyectos educativos, tienen todas por objetivo formar alumnos que continúen estudios superiores, particularmente universitarios. Sin embargo, la práctica en cada uno de estos colegios es sensiblemente diferente, lo cual se ve reflejado en los discursos. Así, mientras en los colegios A y B se inculca a los alumnos y la comunidad escolar que la universidad es la única meta a alcanzar, los colegios C y D matizan este objetivo y abren la puerta a otras alternativas de continuación de estudios para sus alumnos:

"La motivación por la universidad es una evolución natural para un alumno de este colegio, en la que no influimos solo nosotros sino sobre todo los padres. Lo que sí hace nuestro colegio es que trabaja con los alumnos desde pequeños para prepararlos de la mejor manera para el mundo 
universitario y para que sepan tomar decisiones con autonomía y responsabilidad. Por lo demás, los alumnos saben que la PSU no es la única vía de ingreso a las universidades que tienen, puesto que el Bachillerato Internacional también les otorga esta posibilidad”. (Director colegio A).

En el colegio A, la institución recoge la aspiración unánime de los apoderados por un futuro universitario para sus hijos y les otorga distintas vías de acceso a las universidades. En el colegio B, en tanto, cuyo norte — claramente establecido— también es la universidad, pero que cuenta con una composición social mucho más heterogénea que la del colegio A, destacan las posibilidades que un colegio de estas características ofrece para los alumnos menos favorecidos desde el punto de vista socioeconómico:

"Había una idea de que el colegio tenía que educar para la vida, que el niño sea feliz y todo el cuento. Entonces yo digo no, el colegio tiene una obligación y si este se define como científicohumanista, tiene que poner la mayor cantidad posible de alumnos en la universidad". (Director colegio B).

En los colegios C y D, en cambio, no se fomenta únicamente la vía de continuación de estudios universitarios. En el colegio $\mathrm{C}$ se considera, por un lado, que la responsabilidad de la familia es fundamental y prioritaria en la orientación futura de sus hijos y, por otro lado, que muchos alumnos no tienen las capacidades o habilidades (de distinto tipo) para ingresar a la universidad, por lo que asumen como natural la opción de fomentar estudios superiores en otro tipo de instituciones:

"Tenemos varios perfiles de alumnos. Hay niños que quieren ir a la universidad, otros se quieren ir al rubro técnico porque es menos tiempo y es más fácil, van a conseguir más luego un trabajo remunerado porque es lo que falta en sus casas, y hay otro grupo que definitivamente le interesan las fuerzas armadas, carabineros, porque ven a través de esas instituciones una mayor estabilidad laboral. Desde primero básico uno ya vislumbra para dónde van los niños. Entonces en octavo ya sabemos quiénes pueden ir a la universidad y quiénes no". (Directora colegio C).

"Este colegio está inserto en una población donde yo siento que hay muchas habilidades para técnicos, sin embargo la aspiración aquí es una sola: la universidad. Pero cada profesor sabe que hay niños que no son para la universidad, y entonces los orientamos, no de manera pública, hacia otros caminos, desde octavo básico. Después de eso algunas familias se retiran del colegio y van a la educación técnico-profesional". (Orientadora colegio C).

En el colegio D también se minimiza el rol que pueda jugar la institución escolar y se considera que, al recibir un alumnado que viene con serias deficiencias de formación académica desde la enseñanza básica y que muchas veces no presenta "condiciones" para seguir estudios universitarios, es conveniente orientar a los alumnos hacia otras alternativas:

"El mensaje general es que hay que seguir estudiando, no quedarse con cuarto medio, pero no necesariamente en la universidad porque hay alumnos que no tienen las condiciones (...) la universidad no es para ellos, son más prácticos, no tan intelectuales”. (Profesora colegio D).

\subsubsection{La preparación y exigencia académica del colegio}

Uno de los aspectos que todos los autores coinciden en destacar en un colegio que asume estrategias institucionales tendientes a favorecer el acceso de sus alumnos a la 
universidad es contar con una preparación académica adecuada (Hossler, Schmit y Vesper, 1999; McDonough, 1997; Perna, 2006). Aquí también las percepciones son variadas entre los cuatro colegios. Si en los colegios A y B sienten que la preparación es adecuada y la exigencia académica elevada, se aprecia lo contrario en los colegios C y D:

"La exigencia académica es alta (...) es un colegio de corte academicista donde el que no cumple con ciertos criterios debiera pensar en ser reorientado". (Orientadora colegio A).

"Los colegios de esta congregación se caracterizan fundamentalmente por el tema de la excelencia, entonces había que posicionarlo en el mercado y en la opinión pública. El desafío era aumentar los resultados académicos en un corto tiempo (...) yo recibí este colegio con 520 puntos PSU y hoy día tenemos 620 puntos. Es un colegio muy exigente”. (Director colegio B).

"La exigencia no depende solo del colegio o de los profesores. Depende de la materia prima con la que se trabaja. Hay cosas que no dependen de uno". (Orientadora colegio C).

"El colegio no es muy exigente. De repente hay facilidades, si no hiciste algo te dicen 'ya igual te voy a poner un 7". (Presidente del Centro de Alumnos colegio D).

Tal vez uno de los aspectos más llamativos dice relación con el alineamiento que se pudo apreciar en los colegios A y B por parte de los profesores en torno al proyecto educativo institucional (dentro del cual la exigencia académica ocupa un rol preponderante), a diferencia de lo que ocurre en los otros colegios, donde ni el cuerpo docente ni la dirección parecen compartir una visión unificadora al respecto. De hecho, en los colegios C y D se insiste en individualizar las responsabilidades, ya sea en el profesor o en el alumno, pero no en una estrategia o visión de establecimiento.

\subsubsection{La fase de búsqueda de información}

Esta fase del modelo de Hossler y Gallagher (1987) se vislumbra, según estos mismos autores, como la más propicia para la intervención de los establecimientos educacionales, a través de una multitud de alternativas, para informar a los alumnos sobre las distintas posibilidades de estudios universitarios y orientarlos.

Los dispositivos institucionales para informar a los alumnos respecto de las ofertas de instituciones y carreras universitarias o, más ampliamente, de estudios superiores, son en efecto muy variados, y van desde charlas informales entre profesores y alumnos hasta visitas a instituciones específicas u organización de ferias educacionales en los colegios con participación de diferentes instituciones de enseñanza superior.

En el colegio A, en primer lugar, se asume el proceso de orientación como personalizado, por lo que el departamento de orientación realiza un seguimiento individual de los alumnos durante los cuatro años de enseñanza secundaria. Por otro lado, entre la gran cantidad de acciones de información que realizan (visitas a universidades, ferias educacionales dentro del establecimiento, búsqueda asistida por los diferentes sitios web que ofrecen información, etc.) una de las medidas más efectivas, según expresaron sus directivos, es hacer venir al colegio a alumnos universitarios - principalmente exalumnos del colegio- para que expliquen a los alumnos de secundaria en qué consisten, por un lado, las carreras que cursan y, por otro lado, lo que implica transitar desde la enseñanza secundaria a la enseñanza superior:

"[...] la información es excelente. En el colegio había talleres en donde habían alumnos de las mismas carreras que nos daban charlas y de distintas universidades". (Exalumno colegio A). 
En el mismo sentido, aprovechan la variedad de profesiones ejercidas por los apoderados para que estos dicten charlas a los alumnos sobre los desafíos de determinadas profesiones. Con esto, vinculan la decisión sobre estudios universitarios al mundo laboral con el que los alumnos se van a encontrar posteriormente.

Alumnos, apoderados, directivos, profesores y, en general, la comunidad educativa del colegio A coinciden en señalar que los alumnos se encuentran muy acompañados en cuanto al proceso de orientación para seguir estudios superiores y que la cantidad de información que se canaliza a través de distintas vías es ampliamente suficiente.

En el colegio B, que también adopta una amplia gama de dispositivos de información y orientación, ocurre algo muy similar, con la diferencia de que, por la gran cantidad de alumnos que escolarizan, el proceso de orientación es menos personalizado. Además, la participación de los apoderados en este proceso es más marginal, por lo que el colegio asume como su entera responsabilidad informar a sus alumnos sobre las opciones de estudio y orientarlos. Los alumnos y exalumnos de este colegio hacen hincapié en el rol activo de los profesores en el proceso de información y orientación, en estrecha coordinación con el departamento de orientación, así como la relevancia que adquieren las ferias vocacionales realizadas por el colegio:

"Hacen muchas ferias vocacionales. Llega un punto en que uno dice ;oh no! ¡Otra más! Es muy seguido. Podemos decir que estamos bastante informados del tema. Creo que alguien que sale de este colegio y dice me equivoqué, no puede decir que es porque no sabía, porque el colegio siempre nos está informando sobre este tema". (Presidente del Centro de Alumnos colegio B).

En este colegio, el proceso de orientación se inicia temprano en la vida escolar del alumno, a fines de la enseñanza básica. Sin embargo, es en segundo año de enseñanza secundaria cuando el colegio realiza un trabajo profundo y focalizado para preparar a los alumnos, antes de que estos deban optar por un plan diferenciado. El equipo de orientación ha diseñado un programa que tiene una triple vertiente. Por un lado, se hace hincapié en el autoconocimiento, por otro lado se trabaja en las aptitudes y las habilidades académicas del alumno, y finalmente se realiza una proyección del desarrollo de los estudios superiores y profesional.

La lógica seguida por el colegio $\mathrm{C}$ es diferente. A pesar de que también se realizan visitas a universidades, se abren las puertas del establecimiento para que distintas instituciones de educación superior realicen charlas y se programan visitas de exalumnos para que relaten sus experiencias en la universidad $\mathrm{u}$ otras instituciones, la diferencia en relación a los colegios anteriores es que consideran que la mayor responsabilidad en cuanto a la búsqueda de información recae en los alumnos:

"En relación al sitio www.mifuturo.cl $l^{5}$, nosotros les decimos que existe, que se metan, algunos lo hacen, los que se auto gestionan, los que son busquilla, pero el colegio no los guía en ese sitio [...] yo creo que la información nunca es suficiente, pero entregamos lo elemental". (Orientadora colegio C).

El sitio www.mifuturo.cl es un sitio web dependiente del Ministerio de Educación de Chile, muy completo, que presenta amplia información actualizada respecto a los tipos de instituciones existentes en Chile para seguir estudios superiores, las alternativas de financiamiento a las que un alumno puede optar y la empleabilidad de cada carrera, entre otros. 
A pesar de ello, para muchos alumnos los dispositivos establecidos por el colegio son fundamentales, básicamente porque son la única alternativa que tienen de acceder a información a la que, de otro modo, no tendrían acceso.

En el colegio D los directivos reconocen que el trabajo que realizan en cuanto a información y orientación es escaso y que están, en ese sentido, en deuda con los alumnos. Una multitud de factores explican, según los directivos, el escaso impacto de los dispositivos de orientación, entre los que destacan la condición socioeconómica y cultural de los alumnos. Existe la sensación de que cualquier programa serio de información y orientación que se realice en torno a los estudios universitarios cae al vacío, ya que el receptor (en este caso el alumno) no se encuentra preparado para recibir esta información:

"Creo que debemos dejar de concentrarnos en la educación universitaria y hacer más énfasis en la educación técnica. Nuestros alumnos, pienso yo, están mejor preparados para ello. Estamos trabajando con una cantidad de alumnos vulnerables que cada vez es mayor, con problemas personales, económicos, familiares, y por lo tanto no se comprometen con lo que es su educación. No tienen la responsabilidad ni tampoco tienen los aprendizajes previos y por lo tanto no tienen las herramientas para soportar la carga de lo que es una carrera universitaria". (Orientador colegio D).

\subsubsection{La fase de elección: las complejidades de la decisión final}

Según McDonough (1997), la etapa final del proceso de elección de estudios, es decir, el momento específico en el que un alumno debe decidir a qué universidad y a qué carrera va a postular, es una etapa en la que los alumnos se enfrentan a una decisión sumamente compleja. El emplazamiento físico de una institución, las alternativas de financiamiento de los estudios, las percepciones en cuanto a prestigio y calidad de la universidad, el flujo de informaciones recibido en la etapa de búsqueda y los consejos familiares, entre tantos otros criterios, confluyen para que el alumno tome una decisión final. Para otros autores (Ball et al., 2001) la elección final de una institución es más que nada "una cuestión de gustos". Ellos ven la elección de una universidad como la culminación de un proceso de decisión que permitirá a los alumnos seguir encontrándose con los individuos que comparten características similares, sobre todo desde el punto de vista socioeconómico. En nuestro estudio pudimos comprobar que esta decisión se encuentra cruzada por una multitud de variables y factores, más allá de los consabidos costos financieros asociados a los estudios, que en Chile son muy elevados. Por ejemplo, el apoyo que el alumno encuentra en su entorno cercano a un proyecto de estudios, o las percepciones en torno a la capacidad que tengan, en definitiva, de enfrentar determinado tipo de estudios. Queremos dar cuenta a continuación de dos de los criterios que más se repitieron en relación a la decisión final: el primero, una cierta lucha interna que se genera en los alumnos entre la vocación por una carrera y la rentabilidad futura de las carreras. En segundo lugar, la importancia de la prueba de selección universitaria en esta decisión y la manera en que los resultados asociados a ella condicionan la elección de parte del alumno.

\subsubsection{Vocación y rentabilidad de una carrera}

Uno de los temas más sensibles abordados en las entrevistas, en particular con los alumnos, fue el de las razones de fondo que explican el querer elegir —o haber elegidodeterminada carrera en una universidad específica. La respuesta inicial siempre fue la 
vocación. Pero esta vocación, una vez que se avanza en la conversación, se encuentra matizada, a veces muy fuertemente, por aspectos sumamente prácticos — como el costo de los estudios- o de proyección racional del futuro laboral, que no siempre son coincidentes con la declaración inicial de vocación.

Señalan exalumnos de los colegios entrevistados:

Pensé en estudiar Ciencias Políticas, pero abandoné la idea por temas de futuro, por proyecciones, por el campo laboral. Ahí, en esas decisiones influyeron mucho mis papás. Es que mi papá estudió leyes, mi mamá estudió leyes, mi hermana estudia leyes y tienen muy mala apreciación sobre los cientistas políticos. Mi papá decía que era como hacer la pega de un abogado pero en menor medida, que les pagan menos, que no tienen trabajo. (Exalumno colegio A).

A mis papás les interesaba que yo pudiera estudiar una carrera que fuera rentable, daba lo mismo cuál fuera, me dejaban escoger lo que yo quisiera. Pensé en Medicina un tiempo pero al final opté por Ingeniería Civil Química. En algún momento pensé en estudiar Licenciatura en Química, para ser profesor, porque me gusta mucho la química, y me dijeron que no. Me dijeron que estaba bien que me gustara la química, pero entonces que eligiera una ingeniería que tuviese química. (Exalumno colegio B).

Lo que estos exalumnos de los colegios A y B indican, con prístina claridad, es que la influencia de los padres en la decisión final fue muy fuerte y definitivamente marcó el camino a seguir. Así como ellos, son muchos los alumnos que no saben decir a ciencia cierta si lo que están estudiando o van a estudiar es un deseo propio o inducido. Y en esta decisión, la futura rentabilidad económica de la carrera elegida es un aspecto clave. Los adultos entrevistados en los diferentes colegios así lo reafirman:

El tema de la elección de la carrera y universidad pasa por un tema de familia, es una decisión que no la toma autónomamente el alumno. Hay un involucramiento en ese sentido de los padres que siempre están diciendo - me gustaría que estudiaras esto o me gustaría que estudiaras lo otro- y en algunos casos los chicos por darle el gusto a sus familias toman ciertas decisiones. Ven la parte económica, porque es un análisis crudo, más que la vocación. (Directora colegio D). Los papás aspiran a que los hijos sean más que ellos. Si la mamá es enfermera quiere que su hijo sea médico. Si el papá es constructor civil quiere que su hijo sea ingeniero civil. Siempre hay un tema aspiracional en ellos en que de una u otra forma, en esta cultura, el papá se ve muy reflejado en el hijo, entonces le carga cierta emocionalidad al hijo y ciertas aspiraciones que él no pudo concretar. Yo creo que el alumno y sus familias muchas veces deciden en términos económicos. Sería iluso pensar que los cabros hacen una apuesta en un sentido más vocacional (Director colegio B).

\subsubsection{La incidencia de los resultados de la Prueba de selección universitaria (PSU)}

Una de las características del proceso de admisión a las universidades chilenas es que el puntaje obtenido en la prueba de selección universitaria determina las carreras a las que se puede optar. Por lo demás, el tiempo que transcurre entre la entrega de los resultados en esta prueba y la solicitud de admisión es muy breve, de solo algunos días. Esto implica haber reflexionado con anterioridad en relación a la decisión final y, además, haber concebido un plan alternativo en caso de que las condiciones no sean las que el alumno imaginó en un comienzo. 
En muchos casos, el puntaje obtenido en la prueba cierra las puertas de todas las opciones deseadas:

La PSU te limita, sobre todo para los alumnos de colegios municipales. Quizás tengas que estudiar una carrera que nunca quisiste, pero el puntaje PSU te dice que eres bueno para eso. (Exalumna colegio D).

Uno puede postular a 12 carreras como máximo y yo las rellené todas, y quedé seleccionada en mi cuarta preferencia, Diseño Industrial. La primera preferencia era Nutrición. Finalmente tomé la decisión de no inscribirme y prepararme un año más en preuniversitario para volver a dar la prueba. (Exalumna colegio C).

Pero además, el puntaje obtenido en la prueba condiciona la decisión que se debe tomar en muy pocos días, dando origen, por un lado, a decisiones apresuradas para aquellos alumnos que no obtuvieron el puntaje necesario para cursar la carrera que querían, o, como lo atestigua el exalumno del colegio $\mathrm{B}$, pone en marcha verdaderas maquinarias de marketing universitario que intentan captar para las distintas instituciones a los alumnos con altos puntajes, por los beneficios económicos y de prestigio que estos le generan a las universidades, para lo cual realizan ofrecimientos a los que los jóvenes y sus familias difícilmente se pueden negar:

El mismo día que se publicaron los resultados, mi teléfono sonó todo el día. Me llamaron de muchas universidades, y a todas les iba diciendo que no porque mi idea era estudiar en la universidad $X^{6}$. Hasta que fui a la universidad $X$ a preguntar qué beneficios me ofrecían por ser puntaje nacional y cuando me dijeron que no ofrecían nada entonces me lo pensé. Al día siguiente seguí recibiendo llamados, iba en la micro y me llaman de la universidad $Z$ y me ofrecieron todo. Me pagan la carrera completa, me pagan la pensión, todas las comidas diarias, me ofrecen pasajes para ir a ver a mi familia a Concepción dos veces al semestre, y cuando termine la carrera, me ofrecen un posgrado en la misma universidad. Era difícil rechazar algo así y en ese momento decido partir a Valparaíso. La única condición es que no puedo reprobar ningún ramo y debo mantener un promedio sobre 7. (Exalumno colegio B).

\section{DISCUSIÓN}

A través de este estudio se quiso comprender de manera general en qué medida la presencia o ausencia de una cultura institucional de promoción de estudios universitarios en el establecimiento secundario influencia las aspiraciones y decisiones de los alumnos en cada una de las etapas del proceso de elección de estudios. Esta investigación confirma los resultados de estudios realizados en otras latitudes (Hossler et al., 1999; McDonough, 1997), en el sentido de comprender las decisiones que toman los alumnos en cada etapa como fruto de influencias poderosas provenientes tanto del entorno familiar y social como del propio establecimiento educacional. Ahora bien, se puede afirmar que esta amalgama entre familia o clase social de origen y establecimiento educacional se verifica de manera diferente en cada etapa del modelo, así como según las características del colegio y el público que escolariza.

\footnotetext{
Por motivos de confidencialidad, se omiten los nombres reales de las universidades.
} 
De esta manera, en la etapa de predisposición, la inmensa mayoría de los alumnos provienen desde su hogar, cualquiera sea la condición socioeconómica de este, con aspiraciones mayoritarias de estudios universitarios. El nivel de convicción de estas aspiraciones universitarias, sin embargo, es distinto en los colegios que escolarizan a alumnos provenientes de sectores medio-altos y los colegios de sectores medio-bajos. En el primer caso, el colegio no hace más que reforzar aspiraciones que están firmemente arraigadas en las familias y sus entornos. En el segundo caso, se observa en esos colegios una cierta resignación en relación al "tipo de alumno" que reciben, como si estos no tuvieran las capacidades requeridas para seguir estudios universitarios. En ese sentido, se observa que los colegios desaprovechan una oportunidad de romper los mecanismos de perpetuación de la reproducción social y desigualdad en educación.

La etapa de búsqueda de información fue, tal como lo indican los creadores del modelo, la etapa que más se presta para que el colegio intervenga abiertamente en informar y orientar a sus alumnos. El estudio comprueba que se utilizan diversos dispositivos, en todos los colegios. No se identifica ningún colegio en el que no se informe a los alumnos en relación a las posibilidades de estudios superiores. En cambio, se puede apreciar que así como los dispositivos de orientación se encuentran definidos y aplicados por toda la comunidad educativa en los colegios A y B, con un seguimiento intensivo de cada alumno, en los colegios $\mathrm{C}$ y $\mathrm{D}$ existe una distancia enorme entre lo que proponen sus planes de desarrollo institucionales y las prácticas seguidas. En este punto, la noción de habitus institucional cobra todo su sentido, ya que más allá de lo que los proyectos educativos señalen, son las percepciones subjetivas asumidas por los miembros de la comunidad educativa — principalmente profesores, directivos y orientadores-, las que influencian las aspiraciones y actitudes de los alumnos.

En la etapa final del modelo, la decisión final, se observa que esta se encuentra cruzada por un cúmulo de influencias recibidas por parte del entorno cercano del alumno y del establecimiento educacional, pero que, en el contexto chileno, predomina, in fine, la influencia familiar. Las características estructurales de la enseñanza universitaria en Chile obligan al alumno a realizar una transacción con aquellos que van a financiar sus estudios. En el mejor de los casos, los deseos de padres y alumnos coinciden, pero esta situación no es la más común. Siguiendo a Ball et al. (2001), quienes señalan que el proceso de elección de estudios superiores se halla en el seno de dos registros de sentido y de acción, se puede señalar que el registro cognitivo o racional, que dice relación con la adecuación entre los resultados académicos y la selección en una institución, es en Chile fundamental.

En relación a las hipótesis específicas de este estudio, puede decirse, en primer lugar, que la presencia o ausencia de una cultura institucional de ir a la universidad parece efectivamente limitar o ampliar el universo de posibilidades de elección de estudios superiores de un alumno. Esta investigación es coincidente con lo que diversos autores señalan en contextos diversos, es decir, que los recursos formales y las normas organizacionales que el establecimiento pone a disposición de los alumnos en torno a la transición hacia la enseñanza universitaria influencian fuertemente las posibilidades de los alumnos, lo cual es particularmente cierto para los alumnos provenientes de entornos poco familiarizados con el mundo universitario. Si bien no se puede evaluar el peso relativo de los recursos formales a disposición de los alumnos (preuniversitario en el colegio, clases de preparación para la universidad, charlas de exalumnos, visitas a instituciones universitarias) y de las normas organizacionales, a partir de los resultados de este estudio 
se afirma que el aspecto clave que diferencia una cultura institucional de otra y, por lo tanto, el impacto sobre sus alumnos, está dado no tanto por los recursos disponibles sino por las percepciones subjetivas de la comunidad educativa (expectativas sobre el rendimiento académico de los alumnos, ideas preconcebidas sobre ciertas habilidades más desarrolladas que otras en los educandos, etc.), que se traducen en acciones específicas en relación a sus alumnos. En este sentido, cabe destacar y resaltar la particularidad del colegio B, que pese a escolarizar a una población heterogénea, y por lo tanto proveniente de diferentes contextos socioeconómicos y culturales, vehicula un discurso institucional de altas expectativas en torno a las posibilidades de estudios universitarios de sus alumnos, el cual se traduce en acciones concretas.

Esta investigación se propuso también comprobar una segunda hipótesis, es decir, si la cultura institucional de ir a la universidad en un establecimiento escolar influencia a los alumnos con más claridad en las etapas de predisposición y búsqueda de información que en la etapa de elección final, siguiendo el modelo de Hossler y Gallagher (1987). La evidencia presentada en este estudio permite señalar que la cultura institucional tiene un rol relevante en la primera etapa y fundamental en la segunda. En la etapa final, la influencia de esta es menos perceptible que la influencia de la familia y entorno cercano.

Finalmente, cabe destacar que los contextos nacionales influyen fuertemente en la manera en que las culturas institucionales de ir a la universidad van a pesar sobre las decisiones finales de los alumnos. No cabe duda de que las estrategias, expectativas, discursos y recursos de los establecimientos educacionales juegan un rol muy relevante en el proceso de toma de decisiones de estudios superiores de los alumnos chilenos. Sin embargo, más que en otros contextos nacionales, el acceso a la educación universitaria se encuentra aún muy condicionado por factores externos, que los alumnos y sus familias están obligados a considerar. La importancia de la prueba de selección universitaria, en este sentido, es patente, y cruza todo el proceso de decisión. En efecto, un alumno que tenga predisposición para estudiar en la universidad, se inscribe - de manera mayoritaria- en un preuniversitario, institución paralela a los colegios, con fines de lucro, y cuyas relaciones con algunos establecimientos educacionales se tejen a través de vías diversas, siendo el profesorado que labora en ambas instituciones, una de las principales. En la etapa de decisión final, los puntajes obtenidos en la prueba de selección universitaria son los que van a abrir o cerrar las puertas a los alumnos de las carreras deseadas. En esta decisión, como lo vimos, el prestigio de la universidad y el retorno económico de la carrera son factores decisivos para la postulación que los alumnos van a realizar. Y así como este carácter mercantilizado de la educación universitaria chilena implica una limitación de los horizontes posibles, puede significar para otros alumnos una lluvia de ofertas de parte de distintas instituciones, que para la mayoría de estos es imposible rechazar, aunque esto implique cambiar de opción de estudios.

En conclusión, este estudio aporta cierto apoyo a la teoría de McDonough (1997), en el sentido de que el habitus institucional sería un mecanismo de mediación a través del cual el origen socioeconómico y una conciencia de clase influencian el proceso de elección de estudios y por esta vía, contribuye a la reproducción social. En efecto, observamos que son los estudiantes que frecuentan los colegios menos favorecidos desde el punto de vista socioeconómico los que precisamente se encuentran con habitus institucionales menos propicios a la continuación de estudios universitarios.

Otro elemento muy relevante dice relación con las estrategias específicas e intencionadas de promoción de los estudios universitarios (Draelants y Ardoisenet, 2011; Hill, 2008). En 
este sentido, si bien los resultados de este estudio sugieren, una vez más, que la segregación escolar por motivos socioeconómicos que sufren las escuelas chilenas perpetúa la reproducción social, al mismo tiempo, se aprecia la potencialidad del establecimiento para revertir, en parte, esta situación, a través de estrategias intencionadas de promoción de los estudios universitarios, como queda de manifiesto en el colegio B.

\section{REFERENCIAS BIBLIOGRÁFICAS}

Atkinson, W. (2011). From Sociological Fictions to Social Fictions: some Bourdieusian reflections on the concepts of 'institutional habitus' and 'family habitus'. British Journal of Sociology of Education, 32(3), 331-347.

Ball, S.J., Davies, J., David, M., \& Reay, D. (2001). Décisions, différenciations et distinctions: vers une sociologie du choix des études supérieures. Revue française de pédagogie, 136, 65-75.

Burke, C. T., Emmerich, N., \& Ingram, N. (2013). Well-founded social fictions: a defense of the concepts of institutional and familial habitus. British Journal of Sociology of Education, 34(2), 165-182.

Chapman, D. (1981). A model of student college choice. Journal of Higher Education, 52, 490-505.

Draelants, H., \& Ardoisenet, J. (2011). Le rôle de l'établissement d'enseignement secondaire dans la construction des aspirations d'études supérieures. Les Cahiers de recherche du Girsef, 84, 1-40.

Draelants, H., \& Dumay, X. (2011). L'identité des établissements scolaires. Paris: Presses Universitaires de France.

Hill, L. D. (2008). School Strategies and the "College-Linking" Process: Reconsidering the Effects of High Schools on College Enrollment. Sociology of Education, 81, 53-76.

Hossler, D., \& Gallagher, K. (1987). Studying college choice: A three-phase model and the implication for policy makers. College and University, 2, 207-221.

Hossler, D., Schmit, J., \& Vesper, N. (1999). How Social, Economic, and Educational Factors Influence the Decisions Students Make. Baltimore: The Johns Hopkins University Press.

Hossler, D., \& Stage, F. (1992). Family and high school experience influences on the postsecondary plans of ninth-grade students: A structural model of predisposition to college. American Educational Research Journal, 29, 425-451.

Jackson, G. (1982). Public Efficiency and Private Choice in Higher Education. Educational Evaluation and Policy Analysis, 4(2), 237-247.

Lagos, F., \& Palacios, F. (2008). Orientación vocacional y profesional en colegios de bajo nivel socioeconómico. Calidad en la Educación, 28, 204-243.

Litten, L. (1982). Different Strokes in the Applicant Pool: Some Refinements in a Model of Student College Choice. The Journal of Higher Education, 53(4), 383-402.

McDonough, P. (1997). Choosing colleges: how social class and schools structure opportunity. New York: State University of New York Press.

Perna, L. (2006). Studying College Access and Choice: A Proposed Conceptual Model. In J.C. Smart (Ed.), Higher Education: Handbook of Theory and Research (Vol. XXI, pp. 99-157). Netherlands: Springer.

Plank, S., \& Jordan, W. (2001). Effects of Information, Guidance, and Actions on Postsecondary Destinations: A Study of Talent Loss. American Educational Research Journal, 38(4), 947-979.

Reay, D., David, M., \& Ball, S. (2001). Making a Difference?: Institutional Habituses and Higher Education Choice. Sociological Research Online, 5(4). Recuperado de http://www.socresonline. org.uk/5/4/reay.html 
